\title{
Participación del pequeño productor en los mercados: Caso del distrito Colcha de la provincia de Paruro - Cusco
}

\author{
Vianey Bellota \\ Universidad Andina del Cusco - EP Economía \\ Lugar: Distrito de Colcha - Provincia de Paruro
}

\section{INTRODUCCIÓN}

Una de las actividades económicas más importantes de la Región Cusco es la agropecuaria, debido a la representación mayoritaria en las zonas rurales de sus 13 provincias.

Según el mapa de pobreza de la Provincia de Paruro, el Distrito de Colcha se encuentra en el 'nivel pobre' y 'extremo pobre', por lo tanto, la investigación demuestra la participación que tiene el pequeño productor campesino en el mercado y el nivel de ingresos que tiene por la venta de sus productos agropecuarios.

La producción del pequeño productor es de carácter tradicional extensivo, teniendo como actividad principal la agropecuaria, en su mayoría para el autoconsumo, sin dejar de lado su relación comercial con los mercados para la generación de sus propios ingresos monetarios.

Los mercados determinados en la investigación son los internos y los externos, la primera se realiza dentro del ámbito de la provincia, los precios de los productos agropecuarios son menores a los del mercado externo, sin embargo la segunda son aquellas realizadas fuera de la provincia, quiere decir que son economías semimercantilistas.

El estudio demuestra que los ingresos percibidos por las actividades agropecuarias son de subsistencia, no permitiéndole mejorar su calidad de vida; razón por la cual muchos de ellos suelen migrar a otros lugares o dedicarse a otras actividades económicas complementarias.

El artículo ayudará a sustentar la realidad del pequeño productor para así generar nuevas políticas y estrategias de desarrollo en el Distrito de Colcha de la Provincia de Paruro.

Palabras clave: Pequeño productor, productos agropecuarios, mercados internos y externos, ingreso del pequeño productor y destino de la producción. 


\section{OBJETIVOS}

Investigar, analizar y describir la participación que tiene el pequeño productor en los mercados internos y externos de la Provincia de Paruro.

\section{MÉTODOS Y MATERIALES}

La investigación se dio a través de un estudio de caso descriptivo no experimental, iniciándose con las coordinaciones con el alcalde del Distrito de Colcha con la finalidad de considerar los resultados en los planes y proyectos de desarrollo de dicho distrito. Los pasos se detallan a continuación:

a. Revisión documentaria.- Se revisó fuentes secundarias como: el Plan de Desarrollo Provincial, el Plan de Desarrollo Distrital y bibliografía referente a economía campesina, mercados y ferias.

b. Trabajo de campo.- Para el trabajo de campo se consideró la población total del Distrito de Colcha, que según el Censo del año 2007 asciende a 1335 habitantes, de este total se segmentó para obtener la población efectiva, la misma que considera a los productores agropecuarios en la PEA en 384 pobladores, de ello con la muestra estadística para población finita es de 192 entrevistados.

\section{Cuadro N. ${ }^{\circ}$ 01: Muestra estadística}

\begin{tabular}{|c|c|c|}
\hline POBLACIÓN & MUESTRA \\
TOTAL DEL & POBLACIÓN & ESTADÍSTICA \\
DISTRITO DE & EFECTIVA & PARA POBLACIÓN \\
COLCHA & & FINITA \\
\hline 1335 & & 192 \\
\hline
\end{tabular}

Fuente: Trabajo de campo - elaboración propia mayo 2017 
Los instrumentos utilizados fueron: entrevistas semiestructuradas, guía de grupo focal y guía de observación. Para el primer instrumento se obtuvo la muestra estadística para poblaciones finitas aplicándolo a los productores —que en la mayoría de casos fueron varones en un $60 \%$ y mujeres en un $40 \%$-, puesto que el varón representa al jefe de familia y es quien tiene mayor interacción en el mercado. Para el segundo instrumento se obtuvo - por muestra aleatoria - con 5 grupos conformado por 8 productores; finalmente, la aplicación de la guía de observación fue de forma general en las ferias de la Provincia y el Distrito de Colcha.

c. Sistematización de la investigación.- Luego de la obtención de la información primaria (trabajo de campo) y secundaria (revisión documentaria), se realizó el ordenamiento y vaciado de los instrumentos utilizados, arrojando los resultados descritos en el ítem indicado.

\section{RESULTADOS}

El concepto de la FAO, define:

El productor es una persona civil o jurídica que adopta las principales decisiones acerca de la utilización de los recursos disponibles y ejerce el control administrativo sobre las operaciones de la explotación agropecuaria. El productor tiene la responsabilidad técnica y económica de la explotación [...]. (FAO).

Asimismo, la caracterización del pequeño productor —se toma como referencia a diferentes

autores-, indican que tienen una economía de subsistencia, con un tipo de producción de autoconsumo y mercado, además de poseer un mínimo del recurso tierra y animales; tal como se puede ver en el siguiente cuadro: 
Cuadro N. ${ }^{\circ}$ 02: Caracterización del pequeño productor

\begin{tabular}{|c|c|c|c|c|c|}
\hline UBICACIÓN & $\begin{array}{c}\text { TIPO DEL } \\
\text { PEQUEÑO } \\
\text { AGRICULTOR }\end{array}$ & $\begin{array}{c}\text { CANTIDAD } \\
\text { DE TIERRAS } \\
\text { Y NÚMERO } \\
\text { DE } \\
\text { ANIMALES }\end{array}$ & $\begin{array}{l}\text { NIVEL DE } \\
\text { VIDA }\end{array}$ & $\begin{array}{c}\text { PRODUCCIÓN } \\
\text { DIRIGIDA A }\end{array}$ & AUTOR \\
\hline Sierra & $\begin{array}{l}\text { - Campesinos } \\
\text { - Minifundista }\end{array}$ & $\begin{array}{l}1 \text { a } 3 \text { Has. } \\
11 \quad \text { a } \quad 50 \\
\text { animales } \\
\text { mixtos. } \\
\text { Menos de } 1 \mathrm{Ha} \\
10 \text { animales }\end{array}$ & $\begin{array}{l}\text { Subsistencia } \\
\text { Subsistencia }\end{array}$ & $\begin{array}{l}\text { Autoconsumo y } \\
\text { mercado } \\
\text { Autoconsumo }\end{array}$ & Julio Alfaro \\
\hline Sierra & $\begin{array}{l}\text { Pequeños } \\
\text { campesinos }\end{array}$ & ------------------- & Subsistencia & Autoconsumo & $\begin{array}{l}\text { Orlando } \\
\text { Plaza }\end{array}$ \\
\hline Sierra & $\begin{array}{l}\text { Campesinos } \\
\text { pobres }\end{array}$ & ----- & Bajo & Semimercantil & $\begin{array}{l}\text { Efraín } \\
\text { Gonzales }\end{array}$ \\
\hline Sierra & Campesinos & $\begin{array}{l}\text { Menos de } 5 \\
\text { Has. }\end{array}$ & Autosuficiente & $\begin{array}{l}\text { Autoconsumo } \\
\text { familiar } \\
\text { Mercado }\end{array}$ & $\begin{array}{l}\text { Adolfo } \\
\text { Figueroa }\end{array}$ \\
\hline Sierra & $\begin{array}{l}\text { Pequeño } \\
\text { productor }\end{array}$ & Menos de 0,5 & Limitada & Autoconsumo & $\begin{array}{l}\text { Carmen } \\
\text { Fernández }\end{array}$ \\
\hline $\begin{array}{l}\text { Todos los } \\
\text { países }\end{array}$ & $\begin{array}{l}\text { Pequeño } \\
\text { agricultor }\end{array}$ & - & Subsistencia & $\begin{array}{l}\text { Consumo } \\
\text { Mercado }\end{array}$ & ONU - FAO \\
\hline
\end{tabular}

Fuente: Diversos autores 


\section{MAPA DE POBREZA}

En la provincia de Paruro, el total de pobres representa el 78, $8 \%$, de los cuales el 47,7 \% es pobre extremo; el 31,1\% es pobre no extremo, y el 21,2\% no es pobre.

Como se puede observar, el mayor porcentaje está representado por los pobres extremos. El Distrito de Colcha está representado por el 38,8 \% con extremos pobres y ocupa el cuarto lugar de pobreza a nivel de la Provincia de Paruro.

Estos resultados nos demuestran que las familias tienen una economía de subsistencia.

CUADRO N. ${ }^{\circ}$ 03: POBLACIÓN Y CONDICIÓN DE POBREZA

\begin{tabular}{|c|c|c|c|c|c|c|c|c|}
\hline \multirow[b]{2}{*}{$\begin{array}{l}\text { UBI } \\
\text { GEO }\end{array}$} & \multirow{2}{*}{$\begin{array}{l}\text { DPTO. } \\
\text { PROV./DIST } \\
\text { RITO }\end{array}$} & \multirow[b]{2}{*}{$\begin{array}{c}\text { POBLAC } \\
\text { IÓN } \\
\text { TOTAL }\end{array}$} & \multicolumn{4}{|c|}{ POBRE \% } & \multirow{2}{*}{$\begin{array}{l}\text { COEF.VA } \\
\text { R. } \\
\text { POBREZ } \\
\text { A }\end{array}$} & \multirow[b]{2}{*}{$\begin{array}{c}\text { UBICACIÓN } \\
\text { DE LA } \\
\text { POBREZA }\end{array}$} \\
\hline & & & $\begin{array}{l}\text { TOTA } \\
\text { L }\end{array}$ & $\begin{array}{l}\text { EXTRE } \\
\text { MO }\end{array}$ & $\begin{array}{l}\text { NO } \\
\text { EXTRE } \\
\text { MO }\end{array}$ & $\begin{array}{l}\text { NO } \\
\text { POBR } \\
\text { E }\end{array}$ & & \\
\hline & $\begin{array}{l}\text { Provincia } \\
\text { Paruro }\end{array}$ & 32501 & 78,8 & 47,7 & 31,1 & 21,2 & 0,9 & \\
\hline 81001 & Paruro & 3662 & 57,8 & 24,6 & 33,3 & 42,2 & 2,9 & 813 \\
\hline 81002 & Accha & 4049 & 57,3 & 19,8 & 37,5 & 42,7 & 3,2 & 828 \\
\hline 81003 & Ccapi & 4027 & 87,7 & 54,3 & 33,4 & 12,3 & 1,4 & 79 \\
\hline 81004 & Colcha & 1359 & 71,4 & 38,8 & 32,6 & 28,6 & 3,3 & 468 \\
\hline 81005 & Huanoquite & 5879 & 91,4 & 58 & 33,4 & 8,6 & 1,1 & 35 \\
\hline 81006 & Omacha & 6971 & 97,8 & 82,9 & 14,9 & 2,2 & 1,4 & 2 \\
\hline 81007 & $\begin{array}{l}\text { Paccarectam } \\
\text { bo }\end{array}$ & 2466 & 53,3 & 14,8 & 38,5 & 46,7 & 4 & 980 \\
\hline 81008 & Pillpinto & 1372 & 65,7 & 23,6 & 41,1 & 34,3 & 3,5 & 600 \\
\hline 81009 & Yaurisque & 2716 & 85,7 & 48,3 & 37,5 & 14,3 & 1,9 & 109 \\
\hline
\end{tabular}

Fuente: INEI, Mapa de pobreza provincial y distrital 2014 


\section{POBLACIÓN ECONÓMICAMENTE ACTIVA -PEA- DISTRITO DE COLCHA}

En el Distrito de Colcha el 40,24 \% es PEA ocupada, el 0,59\% es PEA desocupada, el resto no pertenece a la PEA. Cabe aclarar, que dentro de la PEA se encuentra el pequeño productor campesino.

Cuadro N. ${ }^{\circ}$ 04: Actividad Económica de la población - PEA

\begin{tabular}{|l|c|c|}
\hline CATEGORÍAS & CASOS & \% \\
\hline PEA ocupada & 478 & 40,24 \\
\hline PEA desocupada & 7 & 0,59 \\
\hline No PEA & 703 & 59,18 \\
\hline TOTAL & 1188 & 100.00 \\
\hline
\end{tabular}

Fuente INEI - XI Censo de población y VI de vivienda 2007

\section{ACTIVIDADES ECONÓMICAS}

Según los resultados del censo realizado en el año 2007, en el Distrito de Colcha la actividad económica más importante es la agricultura y la ganadería con $79,18 \%$, seguido de la actividad económica no especificada 3,71\%; en tercer lugar se tiene que el 3,51\% de la población tiene como actividad principal el comercio. 
Cuadro N..$^{\circ}$ 05: Población ocupada de 15 y más años de edad- Distrito Colcha

\begin{tabular}{|l|r|r|}
\hline \multicolumn{1}{|c|}{$\begin{array}{c}\text { DEPARTAMENTO, PROVINCIA, ÁREA } \\
\text { URBANA Y RURAL, SEXO Y ACTIVIDAD }\end{array}$} & TOTAL & \multicolumn{1}{c|}{$\%$} \\
\hline Distrito COLCHA & $\mathbf{4 8 5}$ & $\mathbf{1 0 0 . 0 0 \%}$ \\
\hline Agricultura, ganadería, caza y silvicultura (001) & 384 & $79,18 \%$ \\
\hline Industrias manufactureras (004) & 1 & $0,21 \%$ \\
\hline Construcción (006) & 14 & $2,89 \%$ \\
\hline Comercio., reparación de vehículos, automóviles, & 17 & $3,51 \%$ \\
\hline motocicletas, efectos personales (007) & 13 & $2,68 \%$ \\
\hline Hoteles y restaurantes (011) & 2 & $0,41 \%$ \\
\hline Trans., Almac., y comunicaciones (012) & 3 & $0,62 \%$ \\
\hline Admin. Púb. y defensa; p. segur. Soc., Afil. (015) & 19 & $3,92 \%$ \\
\hline Enseñanza (016) & 4 & $0,82 \%$ \\
\hline Servicios sociales y de salud (017) & 3 & $0,62 \%$ \\
\hline Hogares privados con servicio doméstico (019) & 18 & $3,71 \%$ \\
\hline Actividad económica no especificada (021) & 7 & $1,44 \%$ \\
\hline Desocupado (022) & & \\
\hline
\end{tabular}

Fuente: INEI - XI Censo de población y V I de vivienda 2007.

\section{RESULTADOS DEL TRABAJO DE CAMPO}

A. TIPOS DE MERCADO.- El pequeño productor está relacionado con dos tipos de mercados, los que se mencionan a continuación:

- Mercados internos.- Los mercados internos se encuentran en la jurisdicción geográfica de la Provincia de Paruro, hacia donde acuden los productores para la venta de sus productos agropecuarios. En el estudio, este mercado se caracteriza por presentar precios menores a los mercados externos, también existe la presencia mayoritaria de intermediarios.

- Mercados externos.- Los mercados externos se encuentran fuera de la jurisdicción geográfica de la provincia, del mismo modo, los pequeños 
productores acuden a estas ferias para la venta de sus productos agropecuarios. La característica de estos mercados es que presentan los precios un poco más elevados con respecto a los internos, a pesar de tener en su mayoría a intermediarios y muy pocos demandantes finales.

B. TIPOS DE PRODUCTOS.- Los productos ofertados por los pequeños productores son:

Productos Agrícolas.- El Distrito de Colcha ocupa el sexto lugar en volumen de comercialización a nivel de toda la provincia, con 563,62 TM., entre papa, maíz, trigo, cebada, haba y otros, de ello se vende en el mercado externo 535,20 TM (Mercado en Cusco y Sicuani) y 28,42 TM en el mercado interno (ferias de Paruro y Accha).

Cuadro N. $^{\circ}$ 06: Flujo de comercialización agrícola en TM

\begin{tabular}{|c|c|c|c|c|c|c|}
\hline \multirow{2}{*}{ Distrito } & \multirow{2}{*}{$\begin{array}{l}\text { Volumen } \\
\text { de venta }\end{array}$} & \multicolumn{4}{|c|}{ Mercado externo } & \multirow{2}{*}{$\begin{array}{l}\text { Mercado } \\
\text { Interno }\end{array}$} \\
\hline & & Cusco & Acomayo & Sicuani & Livitaca & \\
\hline Accha & 749,00 & 530,00 & & 175,00 & 7,00 & 37,00 \\
\hline Ccapi & 466,22 & 442,91 & & & & 23,31 \\
\hline Colcha & 535,20 & 383,42 & & 151,78 & & 28,42 \\
\hline Huanoquite & 783,16 & 744,00 & & & & 39,16 \\
\hline Omacha & 660,63 & 481,78 & & 141,43 & 4,39 & 33,03 \\
\hline Paccarectambo & 663,21 & 630,05 & & & 4,79 & 33,16 \\
\hline Paruro & 627,41 & 596,04 & & & & 31,37 \\
\hline Pillpinto & 239,27 & & 3,98 & 233,33 & & 11,96 \\
\hline Yaurisque & 517,38 & 491,51 & & & & 25,87 \\
\hline Total & 5274,69 & 4299,71 & & 691,54 & 16,18 & 263,28 \\
\hline
\end{tabular}

Fuente: Plan Vial provincial de la provincia de Paruro al 2021

En la comercialización existen canales directos e indirectos; el primero se da cuando la transacción comercial es de 'productor a consumidor final' y en el segundo de 
'productor a intermediarios', esta última es la que predomina en relación al pequeño productor.

Cuadro N. $^{\circ}$ 07: Lugares de venta de productos agrícolas y pecuarios

\begin{tabular}{|l|l|l|l|}
\hline Productos & \multicolumn{2}{|l|}{ Mercado Interno } & $\begin{array}{l}\text { Mercado } \\
\text { Externo }\end{array}$ \\
\hline & En la & $\begin{array}{l}\text { Ferias del } \\
\text { distrito } \\
\text { comunidad y }\end{array}$ & $\begin{array}{l}\text { Otros lugares } \\
\text { mercado externo }\end{array}$ \\
\hline Agrícolas & $12 \%$ & $10 \%$ & $78 \%$ \\
\hline
\end{tabular}

Fuente: Trabajo de campo - elaboración propia mayo 2017

En la «comercialización interna» de Colcha, se realiza una feria anual con motivo de su aniversario en el mes de abril, sin embargo, las comunidades pertenecientes al distrito acuden en forma semanal y mensual a ofertar sus productos agropecuarios, de igual modo, demandan otros productos de las ferias y mercados de Accha y Paruro. Del $100 \%$ de entrevistados, el $78 \%$ vende sus productos en mercados externos y, el $22 \%$ en mercados internos. Los precios de los productos agrícolas se encuentran menores a los costos de producción, sin embargo, de alguna manera los precios son mejores pagados en los mercados externos, aclarando que en estos mercados existen intermediarios y consumidores finales.

Productos pecuarios.- El Distrito de Colcha ocupa el penúltimo lugar en la producción pecuaria, teniendo una producción total de 337,13 TM de carcasa de ganado vacuno, ovino, porcino; de ello 318 TM de carcasa que se destina al mercado externo y 19,13 TM al mercado interno. Los productos en su mayoría son vacunos y ovinos.

Cuadro N. ${ }^{\circ}$ 08: Flujo de comercialización pecuaria

\begin{tabular}{|l|c|c|c|c|}
\hline \multirow{2}{*}{ Distritos } & Volumen & \multicolumn{2}{|c|}{ Mercado externo } & Mercado \\
\cline { 3 - 3 } de venta & Cusco & Combapata & Interno \\
\hline
\end{tabular}




\begin{tabular}{|l|c|c|c|c|}
\hline Accha & 646,96 & 267,1 & 379,86 & 40,93 \\
\hline Ccapi & 540,33 & 540,33 & & 33,95 \\
\hline Colcha & 318,00 & 167.00 & 151.00 & 19.13 \\
\hline Huanoquite & 711,68 & 711,68 & & 44,20 \\
\hline Omacha & 1077,27 & 485,29 & 591,98 & 60,68 \\
\hline Paccarectambo & 421,06 & 421,06 & & 28,10 \\
\hline Paruro & 515,28 & 515,28 & & 30,90 \\
\hline Pillpinto & 192,38 & & 192,38 & 11,88 \\
\hline Yaurisque & 368,58 & 368,58 & & 23,93 \\
\hline Total & 4792,43 & 3476,71 & 1315,72 & 293,70 \\
\hline
\end{tabular}

Fuente: Plan Vial provincial de la provincia de Paruro al 2021

La venta de ganado se distribuye: a nivel de mercado interno, en las ferias de Accha y Paruro y, a nivel de mercado externo en las ferias de Combapata y el camal del Cusco; el $92 \%$ vende a nivel de los mercados externos y el $8 \%$ en el mercado interno. En los mercados externos, en especial en la feria de Combapata, las ventas lo realizan a intermediarios, quienes destinan los productos pecuarios hacia la ciudad del Cusco.

Cuadro N..$^{\circ}$ 09: Lugares de venta de productos agrícolas y pecuarios

\begin{tabular}{|c|c|c|c|}
\hline \multirow{2}{*}{ Productos } & \multicolumn{2}{|c|}{ Mercado Interno } & $\begin{array}{c}\text { Mercado } \\
\text { Externo }\end{array}$ \\
\hline & $\begin{array}{c}\text { En la } \\
\text { comunidad }\end{array}$ & $\begin{array}{c}\text { Ferias del } \\
\text { distrito y } \\
\text { provincia }\end{array}$ & $\begin{array}{c}\text { Otros lugares } \\
\text { Mercado Externo }\end{array}$ \\
\hline Pecuarios & $5 \%$ & $3 \%$ & $92 \%$ \\
\hline
\end{tabular}

Fuente: Trabajo de campo - elaboración propia mayo 2017

Los precios percibidos por la venta de los ganados son por debajo de sus costos de producción, a pesar de ello, para los pequeños productores significa un ahorro de dinero en semovientes que poseen, especialmente ganado vacuno y estos son utilizados para momentos de déficit presupuestal familiar (periodo escolar, enfermedades, construcción de sus viviendas y otros). 


\section{INGRESOS DEL PEQUEÑO PRODUCTOR PERCIBIDO EN LOS} MERCADOS.- En el trabajo de campo realizado, de 192 productores entrevistados - para el caso de productos agrícolas- el $73 \%$ destina sus producción agrícola al autoconsumo y el 27 \% lo destina al mercado. En cambio, en el caso de productos pecuarios, el $89 \%$ de productores lo destina al mercado, en especial de ganado vacuno y, en segundo lugar el ovino; el $11 \%$ lo destina a autoconsumo, referido solo a ganado ovino y porcino.

Cuadro N. ${ }^{\circ}$ 10: Destino de la producción del pequeño productor

\begin{tabular}{|c|c|c|c|}
\hline $\begin{array}{c}\text { Producción } \\
\text { dirigida }\end{array}$ & Mercados & Autoconsumo & Total \\
\hline Agrícolas & $27 \%$ & $73 \%$ & $100 \%$ \\
\hline Pecuarios & $89 \%$ & $11 \%$ & $100 \%$ \\
\hline
\end{tabular}

Fuente: Trabajo de campo - elaboración propia mayo 2017

Los ingresos percibidos por el pequeño productor de la venta de sus productos son tanto del agrícola como del pecuario. Para el caso de productos agrícolas, estos son vendidos en mercados internos y externos en cantidad promedio de 120 kilos mensuales, esto dependiendo de la temporalidad, entre los productos de papa, maíz trigo y habas, tienen un precio promedio por kilo de S/.1, 10; generando un ingreso de S/.132.00 por mes y S/.1584.00 por año. 


\section{Cuadro $N .^{\circ}$ 11: Ingreso anual de pequeño productor}

\begin{tabular}{|c|c|c|c|c|c|c|}
\hline Productos & $\begin{array}{c}\text { Cantidad } \\
\text { vendida } x \\
\text { mes }\end{array}$ & $\begin{array}{c}\text { Cantidad } \\
\text { vendida } x \\
\text { año }\end{array}$ & $\begin{array}{c}\text { Detalle } \\
\text { productos }\end{array}$ & $\begin{array}{c}\text { Precio en } \\
\text { promedio } \\
\text { S/. }\end{array}$ & $\begin{array}{c}\text { Ingreso } \\
\text { promedio } \\
\text { x mes }\end{array}$ & $\begin{array}{l}\text { Ingreso } \\
\text { x año }\end{array}$ \\
\hline Agrícolas & $120 \mathrm{kls}$ & $1440 \mathrm{kls}$ & $\begin{array}{l}\text { Papa, } \\
\text { maíz, } \\
\text { trigo, } \\
\text { habas }\end{array}$ & $\begin{array}{c}0,70 \mathrm{Kl} \\
1,4 \mathrm{Kl} \\
1.00 \mathrm{Kl} \\
1,3 \\
\text { Precio } \\
\mathrm{X}: 1,10\end{array}$ & 132.00 & 1584.00 \\
\hline Pecuarios & & $\begin{array}{l}1 \text { gan. } \\
2 \text { gan. }\end{array}$ & $\begin{array}{c}\text { Vacuno } \\
\text { Ovino }\end{array}$ & $\begin{array}{l}720.00 \\
130.00\end{array}$ & & $\begin{array}{l}720.00 \\
260.00\end{array}$ \\
\hline \multicolumn{6}{|r|}{ Total S/. } & 2584.00 \\
\hline
\end{tabular}

Fuente: Trabajo de campo - elaboración propia mayo 2017

En la venta de productos pecuarios ocurre lo contrario: el $89 \%$ de productores lo destina al mercado, en promedio 1 cabeza de vacuno y 2 cabezas de ovino por año son vendidos, de ello el precio promedio de las cabezas de vacuno es de S/.720.00 y de S/. 140.00 del ovino, lo que representa un ingreso anual de S/.980.00 por la venta de productos pecuarios.

Por lo tanto, el ingreso total promedio que percibe el pequeño productor pecuario y agrícola es de S/.2584.00 anuales, dicho ingreso es de subsistencia, demostrándose así las características del pequeño productor que dan los diversos autores indicados en el cuadro 02; es decir, no cuentan con capital suficiente ni tecnologías adecuadas. El costo de la mano de obra para realizar labores agrícolas se incrementó debido a la escasez de mano de obra por la migración de jóvenes a la ciudad y, porque estos prefieren trabajar en obras públicas — con honorarios más altos- en los municipios. También influye el hecho que la zona sea accidentada en el distrito y, por último, el elevado precio de los insumos para la actividad agropecuaria y la falta de capacitación de los pequeños productores. 


\section{DISCUSIÓN}

Según (Kervyn, 1988, pág. 92), la agricultura y la ganadería son las actividades fundamentales del productor campesino en la sierra del Perú, estas dos actividades son interdependientes puesto que parte del producto agrícola sirve como insumo para la ganadería y esta, a su vez, proporciona insumos para la agricultura, lo que es conocida como 'economía de escala nula'.

Desde la década de los ochenta se puso en moda la liberalización de mercados, como de las reformas sociales a fines del siglo XX. Los programas de ajuste estructural tienen como objetivo promover la mayor eficiencia y crecimiento económico basado en el pleno funcionamiento del Estado, sin embargo, se conoce muy poco cómo funcionan los mercados en el ámbito rural (Gonzales de Olarte, 2005).

La organización de las comunidades campesinas gira en torno a la tierra, este recurso configura las prácticas y valores culturales; además, en el mundo andino la tierra es concebida como símbolo de fertilidad, a la que se le debe agradecimiento por la vida. (Allpa, 2012).

Los resultados de la caracterización del "pequeño productor" en el Distrito de Colcha, nos muestran que su nivel económico es de subsistencia, razón por la cual según el mapa de pobreza, aún se mantiene en extremo pobre. Sin embargo, debe aprovechar de la mejor manera sus escasos medios de producción, siendo la actividad agropecuaria una fuente de autoconsumo y de mercado, a través de los mercados internos y externos.

En estos últimos 5 años el "pequeño productor" viene sufriendo cambios debido a que la rentabilidad agrícola y pecuaria es baja, razón por la cual dichos productores destinan la fuerza de su trabajo a las municipalidades u otras instituciones que requieran mano de obra, quiere decir que se viene originando la descampesinización (Sic.), (migración del campo a la ciudad, dejando sin habitantes al campo), lo cual amerita una mayor atención en este sector por parte de los gobiernos locales, regionales y nacional.

En esta discusión participó el alcalde del distrito, quién indicó que ante estos resultados generaría un cambio en la producción agrícola por productos que sean más rentables caso la palta, durazno, higo y la quinua. Buscará la especialización productiva de su distrito y no la diversificación esperando contar con un mayor presupuesto para su municipio. 


\section{CONCLUSIONES}

- La investigación usó como método el «Estudio de caso - descriptivo no experimental.»

- La caracterización del 'pequeño productor' se basa en la posesión del recurso tierra, cantidad de semovientes, nivel de vida, destino de la producción e ingresos.

- Los resultados de la investigación demuestran que el 'pequeño productor' del Distrito de Colcha, tiene una participación directa en los mercados internos y externos, donde oferta sus productos agropecuarios a precios ínfimos.

- Los ingresos promedio percibidos por el 'pequeño productor' es de S/.2584.00 anuales y S/. 215.00 mensuales, calificado como 'ingreso de subsistencia'. Por esta razón se viene reflejando la descampesinización (Sic.) en dichos productores, que vienen realizando cambios en el uso de su mano de obra, no para la actividad agropecuaria, sino para trabajos como peones o maestros de obra en diferentes obras del municipio u otras instituciones públicas o privadas, debido a que el salario percibido es mayor.

- Ante estos cambios en el distrito la autoridad municipal tomará nuevas estrategias de cultivo que tengan mayor rentabilidad y sean escasos en el mercado, para que de esta manera mejore sus niveles de ingreso y no trasladar la mano de obra del pequeño productor a otras actividades como subempleadas.

\section{BIBLIOGRAFÍA}

Allpa, G. (2012). Contexto social, económico e institucional de la Región Cusco. Allpa, 32. Obtenido de http://www.allpa.org.pe/sites/default/files/Las\%20Comunidades\%20Campesinas\%20\%20Cusco\%20\%282010\%29_1.pdf

FAO, D. d. (s.f.). http://www.fao.org/docrep/004/x2919s/x2919s05.htm. Recuperado el 2017, de http://www.fao.org/docrep/004/x2919s/x2919s05.htm: http://www.fao.org/docrep/004/x2919s/x2919s05.htm

Gonzales de Olarte, E. (2005). Mercados en el ámbito rural peruano. SEPIA Seminario Permanente de Investigación Agraria, 51. Obtenido de http://www.sepia.org.pe/index.php?fp_cont $=878$

Kervyn, B. (1988). La Economía Campesina en el Perú: Teorías y Políticas. Cusco, Perú: Centro de Estudios Rurales Andinos Bartolomé de las Casas. 